\title{
Molecular Cloning and Chromosomal Mapping of DNA Rearranged with the Parathyroid Hormone Gene in a Parathyroid Adenoma
}

\author{
Andrew Arnold," Hyung Goo Kim, ${ }^{\star *}$ Randall D. Gaz, ${ }^{\ddagger}$ Roger L. Eddy, Yoshimitsu Fukushima, \\ Thomas B. Shows," and Henry M. Kronenberg* \\ *Endocrine Unit and ${ }^{\ddagger}$ Department of Surgery, Massachusetts General Hospital, and Harvard Medical School, Boston, Massachusetts \\ 02114; and ${ }^{\S}$ Department of Human Genetics, Roswell Park Memorial Institute, Buffalo, New York 14263
}

\begin{abstract}
Parathyroid adenomas are common benign neoplasms for which no chromosomal defects have been described. We recently found two parathyroid adenomas bearing clonal restriction fragment abnormalities involving the PTH locus, and now show that in one of these tumors: (a) a DNA rearrangement occurred at the PTH locus; $(b)$ the rearrangement separated the PTH gene's 5' flanking region from its coding exons, conceivably placing a newly adjacent gene under the influence of PTH regulatory elements; $(c)$ the DNA that recombined with PTH normally maps to 11q13, the known chromosomal location of several oncogenes and the gene for multiple endocrine neoplasia type $I$; and $(d)$ the rearrangement was a reciprocal, conservative recombination of the locus on 11 q13 (Human Gene Mapping Library assignment D11S287) with PTH (on 11p15).

These data provide molecular cytogenetic evidence for the clonal occurrence of a major chromosome 11 aberrancy in this benign parathyroid tumor. The D11S287 clone could prove useful in genetic linkage analyses, in determining precise $11 q 13$ breakpoints in other neoplasms, and in identifying a gene on chromosome 11 that may participate in parathyroid tumor development.
\end{abstract}

\section{Introduction}

Chromosomal abnormalities (e.g., translocations, specific deletions) are important mechanisms of neoplasia. Well-characterized examples include the Philadelphia chromosome $t(9 ; 22)$ in chronic myelogenous leukemia, in which the c-abl oncogene is somatically rearranged, and the translocations in Burkitt lymphoma, in which one of the Ig loci (on chromosome 14,2 , or 22 ) is rearranged with the c-myc region on chromosome $8(1,2)$. High resolution cytogenetic methods have greatly facilitated the study of these and other hematopoietic tumors, and cytogenetic study has historically preceded the elucidation of the molecular oncologic basis of such tumors. In addition, cytogenetic analyses have provided important clues in guiding the molecular studies of a limited number of nonhereditary solid tumor types $(3,4)$. A cytogenetic approach has not been possible, however, in the study of parathyroid ade-

Address reprint requests to Dr. Andrew Arnold, Endocrine Unit, Massachusetts General Hospital, Boston, MA 02114.

Received for publication 16 November 1988 and in revised form 3 February 1989.

J. Clin. Invest.

(C) The American Society for Clinical Investigation, Inc.

0021-9738/89/06/2034/07 \$2.00

Volume 83, June 1989, 2034-2040 nomas (or for that matter, of many other solid tumors), and underlying molecular mechanisms of neoplasia in this disease are unknown.

Parathyroid adenomas are examples of benign, clonal neoplastic growth (5). We recently found two parathyroid adenomas with tumor-specific restriction fragment abnormalities involving the PTH locus (5). We now report molecular studies of one of these tumors, which show that $(a)$ this tumor's abnormal PTH restriction fragment represents a clonal DNA rearrangement at the PTH locus; $(b)$ this rearrangement, which in novel fashion involves a locus encoding a major differentiated tissue-specific protein, separates the PTH gene's 5' flanking region from its coding exons (conceivably placing a cellular oncogene under the influence of PTH regulatory elements in the parathyroid cell); $(c)$ the DNA that rearranged with PTH in this tumor normally maps to chromosome 11, band q13, the known location of at least three putative oncogenes and the possible location of the gene for multiple endocrine neoplasia type I (MEN-I) ${ }^{1}$; and $(d)$ the rearrangement constitutes a reciprocal and conservative recombination of the locus on 11q13 with the PTH locus (on 11p15), thus providing molecular cytogenetic evidence for a major chromosomal rearrangement in this (benign) parathyroid adenoma.

\section{Methods}

Patient $Y$ was a 68-yr-old woman in 1981 when she presented with nephrolithiasis, bone pain, and an 11-yr history of hypercalcemia. She had no history of neck irradiation and no family history of endocrinopathy. Evaluation at the Massachusetts General Hospital revealed a serum calcium of $13 \mathrm{mg} / \mathrm{dl}$ ( $3.25 \mathrm{mmol} / \mathrm{liter})$, phosphate of $2.5 \mathrm{mg} / \mathrm{dl}$ ( $0.81 \mathrm{mmol} /$ liter), fourfold elevated PTH, and hypercalciuria (24-h urinary calcium $=329 \mathrm{mg}$ ), all consistent with primary hyperparathyroidism. At neck exploration, a large, 6-g left lower parathyroid adenoma was removed, with an atrophic left upper gland identified and shown to be normal on biopsy. No histologic features suggestive of carcinoma were present. Serum calcium normalized postoperatively; normocalcemia and a normal PTH level were documented 7 yr later, in 1988.

The parathyroid tumor specimen was frozen in liquid nitrogen shortly after surgical removal. Extraction of high molecular weight DNA, restriction enzyme digestion, and Southern blotting were performed as previously described (6). PTH gene fragments used as hybridization probes for Southern blots and genomic library screening were the 775-bp Bgl II fragment ( $5^{\prime}$ PTH probe) and the 2,600-bp Sst IEco RI fragment (3' PTH probe) from pPTHg108 (7). The $b c l-1$ probe was a $2.1-\mathrm{kb}$ Sst I fragment (8) contributed by Dr. Y. Tsujimoto, and the int-2 probes were inserts of plasmids SS6 and BB4 (9), contributed by Dr. C. Dickson.

1. Abbreviations used in this paper: MEN-I, multiple endocrine neoplasia type I. 
For cloning of the 10.5-kb Hind III fragment (see Results), a Hind III library of tumor genomic DNA was constructed in the $\lambda 2001$ bacteriophage vector; the acceptable range of insert size for this vector is 9-22 kb (10). $4 \times 10^{5}$ recombinant phage were screened by hybridization with the $5^{\prime}$ PTH probe. 10 positive plaques were obtained; DNA was extracted from 4 of these purified phage, and 3 contained the appropriately sized insert (the other contained multiple smaller inserts that presumably included a Hind III fragment from the normal PTH allele). As expected, the $5^{\prime}$ PTH probe hybridized strongly to the 10.5 kb insert, while the $3^{\prime}$ PTH probe did not hybridize to this insert on Southern blots of Hind III-digested phage DNA. To clone the 12-kb Bam HI rearranged fragment (see Results), a Bam HI library of tumor genomic DNA was constructed in bacteriophage EMBL3 (11). The acceptable insert size range for EMBL3 is $10-22 \mathrm{~kb}$; thus it was anticipated that the normal $(16 \mathrm{~kb})$ PTH allele would be represented in the library in addition to the abnormal 12-kb insert. Therefore, the library $\left(2 \times 10^{6}\right.$ recombinant phage) was screened with the $3^{\prime}$ PTH probe, and the 20 positively hybridizing phage were further screened with the $5^{\prime}$ PTH probe. 2 of these 20 showed no hybridization to the $5^{\prime}$ PTH probe, and were selected as probably containing the desired insert. The highly preferential cloning of the normal PTH allele (hybridizing to both $5^{\prime}$ and $3^{\prime}$ PTH probes) was apparently due to its more appropriate size as an EMBL3 insert. The two phage putatively containing the rearranged $12-\mathrm{kb}$ insert indeed had inserts of this size, which, by restriction mapping, were identical.

Non-PTH DNA fragments A, B, and C (see Results) were subcloned in pUC-18; the inserts were excised from the vector, agarose gel purified, and ${ }^{32} \mathrm{P}$-labeled (12) for use as hybridization probes.

Chromosomal mapping using human-mouse somatic cell hybrids $(13,14)$, Southern blotting $(15)$, and in situ hybridization $(16,17)$ was performed as previously described.

\section{Results}

Structure and cloning of the rearranged PTH gene. Initial Southern blots of patient Y's DNA indicated that an abnormal Bam HI restriction fragment hybridizing to a PTH probe was present in her parathyroid tumor but not in peripheral blood leukocytes (5). This clonal abnormality was associated with loss of $50 \%$ of the intensity of the band representing the normal PTH gene; thus the abnormal gene, present in roughly equal amounts to the normal gene, was likely to be allelic to the normal gene. Further, this equality suggested that the $a b-$ normality was present in all of the cells of the tumor and thus was likely to have been present in the progenitor cell of the tumor. These properties suggested that the gene abnormality was important pathogenetically. We first sought to characterize this structural abnormality more fully using Southern blots with six additional restriction enzymes and two PTH probes (see Methods). Abnormal restriction fragments hybridizing to the PTH probes were seen in several enzyme digests (Fig. 1). Interestingly, the size of the tumor-specific abnormal fragment differed depending on whether the $5^{\prime}$ or $3^{\prime}$ PTH probe was used with a particular enzyme. In addition, several enzymes (e.g., Eco RI with the $3^{\prime}$ PTH probe) yielded a normal pattern in tumor DNA. A map deduced from this data is shown in Fig. 2 $A$, and indicates that a rearrangement of the PTH locus with other, non-PTH DNA had occurred at one of the two PTH alleles in the tumor. This rearrangement occurred at a breakpoint within the first intron of the PTH gene, separating the coding exons of PTH (II and III) from noncoding exon I. No restriction enzyme tested placed $5^{\prime}$ and $3^{\prime}$ hybridizing PTH sequences on the same sized genomic fragment, and therefore with these data we could not determine whether the parts of the PTH gene were in continuity on one chromosome, or instead had been involved in a reciprocal interchromosomal translocation.

The structure of the rearrangement did suggest a number of hypothetical mechanisms by which it could be functionally relevant in parathyroid tumorigenesis. Among these is the pos-
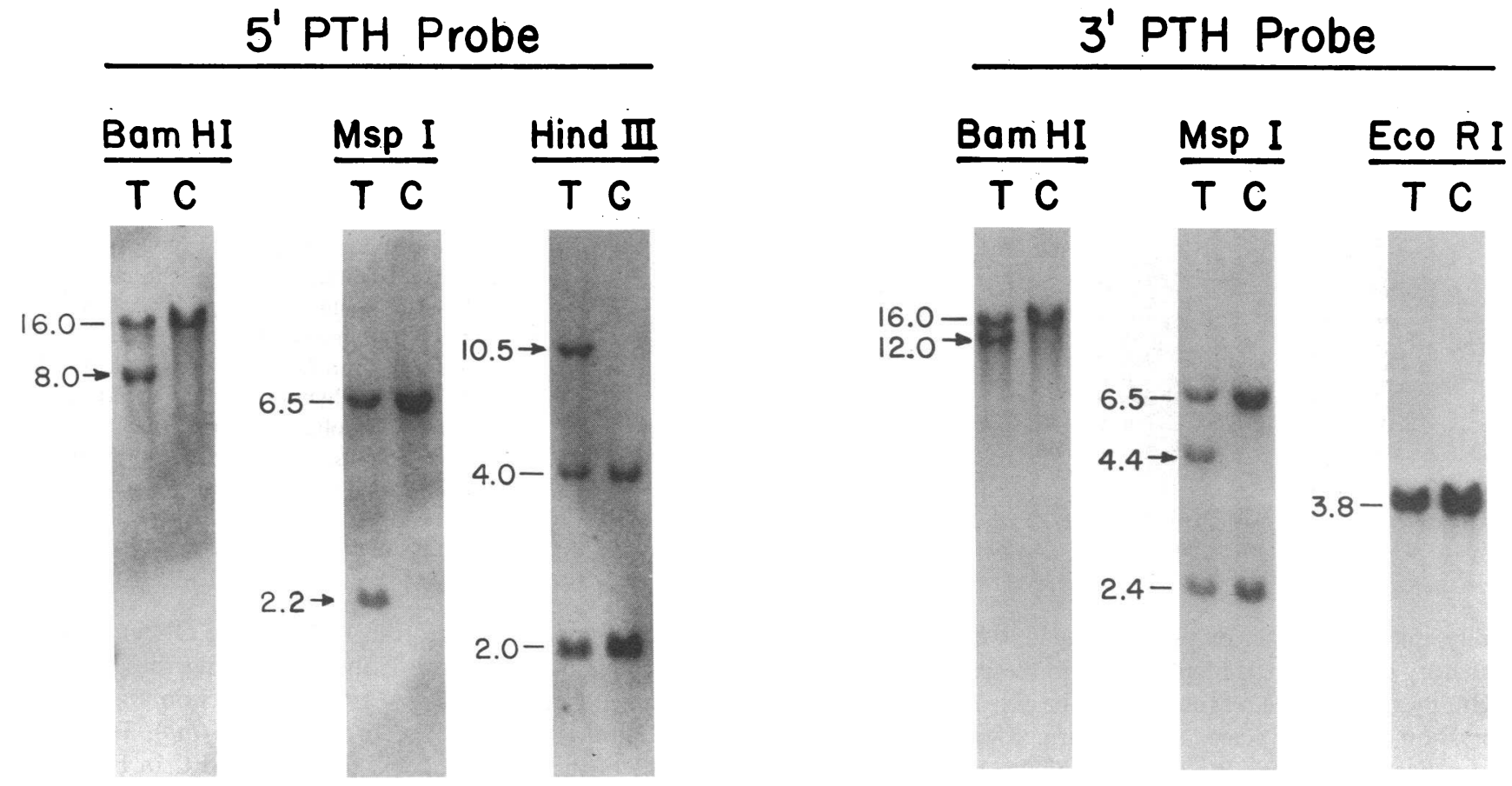

Figure 1. Genomic Southern blot analysis of PTH gene rearrangement in parathyroid tumor Y. Autoradiograms of Southern blots are shown, after digestion of equal quantities of DNA from the patient's tumor (lanes $T$ ) and control leukocytes (lanes $C$ ) with the indicated restriction endonucleases, and probing with radiolabeled DNA fragments from the 5' or 3' part of the PTH gene. The sizes of normal PTH bands (marked with a dash) and rearranged PTH bands (marked with an arrow) are shown in kilobases. 
A

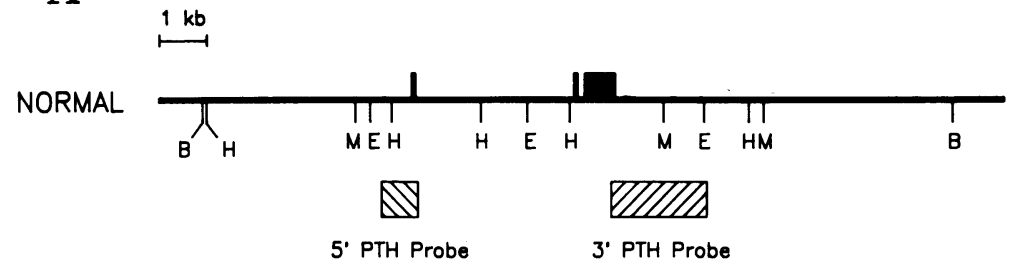

B
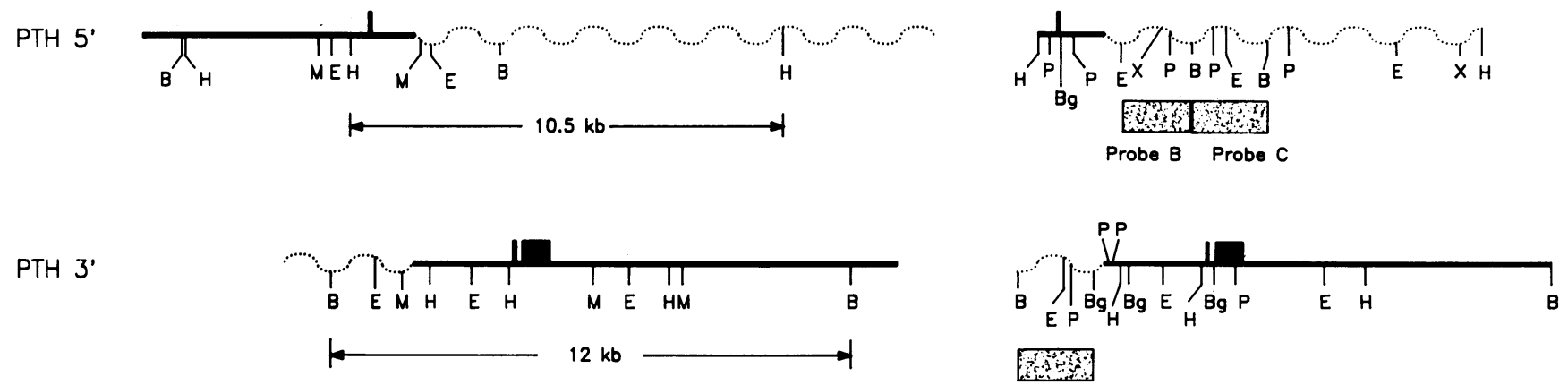

Probe A
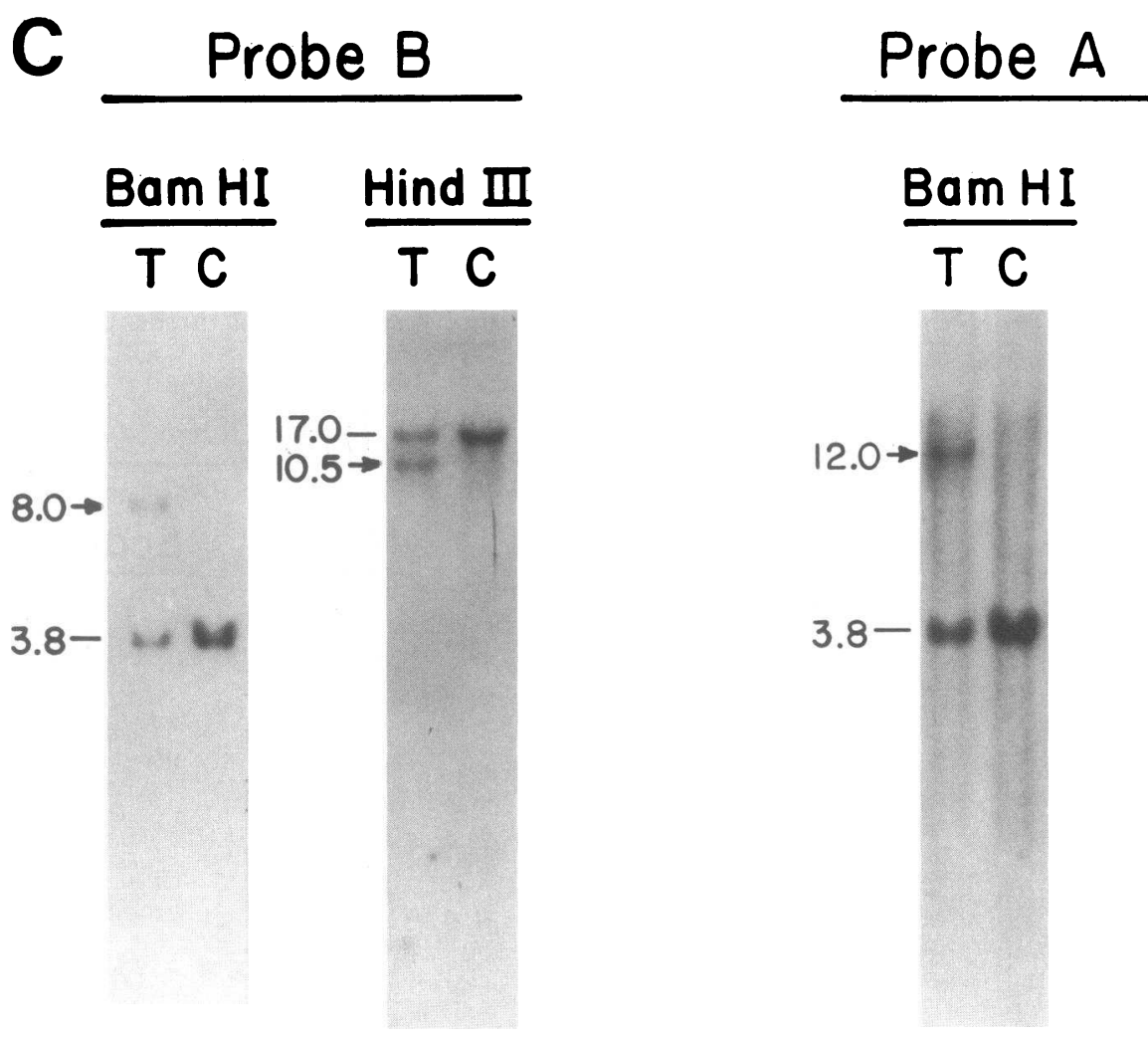

Figure 2. Structure of DNA involved in tumor-specific PTH gene rearrangement. Genomic restriction maps of the normal PTH gene and rearranged PTH-containing segments are shown in $A$, while maps of corresponding cloned bacteriophage inserts (10.5-kb Hind III and 12-kb Bam HI) are shown in $B$. The heavy line denotes PTH sequences, with the PTH exon positions filled in solidly above it. Non-PTH sequences brought adjacent to PTH by the rearrangement are identified by the wavy dotted line. Crosshatched boxes denote the locations of normal PTH fragments used as radioactive probes in these studies, while lightly shaded boxes mark the non-PTH probes. Symbols for restriction sites: B (Bam HI), Bg (Bgl II), E (Eco RI), H (Hind III), M (Msp I), P (Pst I), X (Xho I). No Kpn I sites were present in either cloned insert, and additional Pst I sites exist between fragment $\mathrm{C}$ and the right-hand Hind III site, the number and positions of which remain uncertain. In $C$ the genomic Southern blots from Fig. 1 were stripped of PTH probe and rehybridized with the indicated non-PTH probes. Tumor-specific rearranged bands, identical in size to those in Fig. 1, are marked with arrows, while bands representing normal (unrearranged) alleles are marked with dashes.

sibility that the $5^{\prime}$ part of the PTH gene, which contains a regulatory region that is highly active in the parathyroid cell, might, by virtue of the rearrangement, be able to influence the expression of a newly placed downstream growth-related gene. To further characterize the structure of the DNA that rearranged with the PTH gene in this tumor, we cloned the sequences adjacent to both the $5^{\prime}$ and the $3^{\prime}$ PTH gene segments.

We chose the 10.5-kb Hind III fragment in Fig. $2 A$ (includes $5^{\prime}$ PTH DNA and noncoding exon I) and the $12-\mathrm{kb}$
Bam HI fragment (includes the PTH coding DNA) for this purpose, and bacteriophage clones containing these inserts were selected (see Methods). A detailed restriction map of the 10.5-kb Hind III insert is shown in Fig. $2 B($ top $)$. Two subfragments of this insert, labeled probes $B$ and $C$ in Fig. $2 B$, each containing solely non-PTH sequences, were used as hybridization probes in studies described below. That the bacteriophage insert was a faithful representation of the tumor's abnormal genomic rearrangement was confirmed by the use of 
probe B with the identical blots shown in Fig. $1 \mathrm{~A}$. As expected, a tumor-specific Hind III band was seen that precisely comigrated with that seen with the $5^{\prime}$ PTH probe (Fig. $2 C$ ). Similarly, probe $B$ hybridized with the identically sized $(8 \mathrm{~kb})$ rearranged Bam HI fragment recognized by the $5^{\prime}$ PTH probe in Fig. $1 A$ (Fig. $2 C$ ).

The restriction map of the 12-kb Bam $\mathrm{HI}$ insert is shown in Fig. 2 B (bottom). A 1,650-bp Bgl II-Bam HI fragment, marked probe $A$ in the figure, was used as a hybridization probe in further studies. As expected, probe A hybridized to a Bam HI fragment on the blot from Fig. 1 that precisely comigrated with the abnormal 12-kb band seen with the $3^{\prime}$ PTH probe (Fig. $2 C$ ).

Probes A, B, and C each contained single copy sequence in the normal human genome, based on the limited number (one or two) of hybridizing bands they detected on Southern blots washed at high stringency $\left(0.1 \times\right.$ standard saline citrate, $\left.63^{\circ} \mathrm{C}\right)$. One probe (C) hybridized with 1-3 additional discrete fragments (depending on the enzyme used) when the Southern blot was washed at somewhat lower stringency $(0.1 \times$ standard saline citrate, $\left.50^{\circ} \mathrm{C}\right)$, raising the possibility that the sequence may be part of a family of related genes. In addition, probes $A$ and C (B was not tested) hybridized as a single copy sequence in the mouse genome under the high stringency conditions. These data suggest that the DNA that rearranged with the PTH gene in this parathyroid tumor is highly conserved and might well include the coding sequence of a gene.

We attempted to determine whether probe $A$ (from the clone containing the $3^{\prime}$ PTH region) and probe B (from the clone containing the $5^{\prime}$ PTH region) originated from the same genetic locus, and if so, whether the possibly reciprocal recombination of this region with the PTH gene was conservative. Southern blots of normal human genomic DNA cut with a variety of restriction enzymes were hybridized with probes $A$ and $B$ (separately), chosen because of their proximity to the PTH gene breakpoint in their respective independent bacteriophage clones. Precise comigration of resulting bands was observed for all enzymes (e.g., Bam HI, Hind III, Pst I, Kpn I) studied except those for which probes A and B would be expected to hybridize to different fragments based on the map in Fig. $2 B$ (e.g., Bgl II, Eco RI). For example, probes A and B independently hybridized to a single 3.8-kb Bam $\mathrm{HI}$ fragment in normal human leukocyte DNA samples, including the leukocyte control DNA from patient $\mathrm{Y}$, as shown in Fig. $2 C$. These data constituted strong evidence that fragments $A$ and $B$ are under normal circumstances in the same genomic region that has undergone a reciprocal and highly conservative recombination with the PTH gene in patient Y's parathyroid tumor. A composite restriction map of this unnamed locus, which has now been termed D11S287 (see below), in its normal (unrearranged) state was derived from the Southern blots described above and from the maps of the independent phage clones and subclones (Fig. 3).

Chromosomal mapping of D11S287. The normal chromosomal location of the non-PTH DNA that underwent tumorspecific rearrangement in this parathyroid tumor was sought to (a) elucidate the type of somatic recombination with PTH found in the tumor (i.e., between or within chromosomes), and $(b)$ guide a search for the identity of this DNA, which could be near or part of an oncogene. Probes $A$ and $C$ were used on Southern blots of human-mouse hybrids with defined human chromosome complements (Fig. 4). The results, sum-

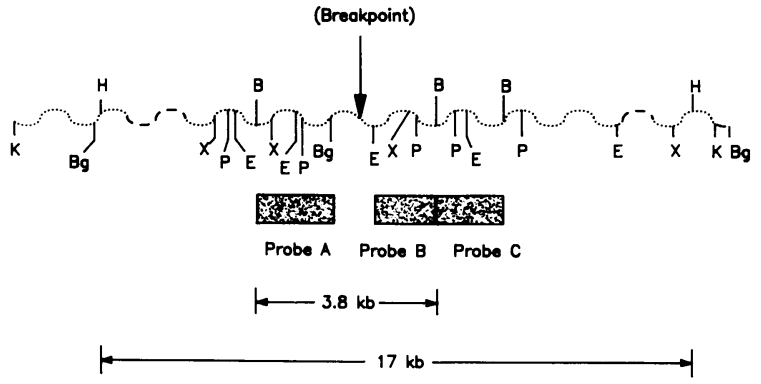

Figure 3. Deduced genomic restriction map of the D11S287 region in its normal (unrearranged) state. This is a combination of the maps derived from the separate use of probe $A$ and of probes $B$ and $C$, justified by hybridization of both probes $A$ and $B$ to these precisely comigrating Southern blot bands: 3.8-kb Bam HI, 2.1-kb Pst I, 2.5-kb Xho I, 17-kb Hind III, and 25-kb Kpn I. Heavy dashed lines denote areas not drawn to scale (compressed), and additional sites could exist in these distal regions for enzymes (e.g., Eco RI) with sites between these regions and the nearest probe. The position at which the D1 1S287 locus was broken in tumor $\mathrm{Y}$ is marked with an arrow. Symbols for restriction enzyme sites are as in Fig. 2, with the addition of K (Kpn I).

marized in Table I, localized the fragments to chromosome 11 in the region from 11 q13 to 11 qter. Probes $A$ and $C$ were used for in situ hybridization studies as well. Both of these sequences were localized to 11q13.3-11q13.5 (Fig. 5, $A$ and $B$ ). The genomic region containing fragments $\mathrm{A}, \mathrm{B}$, and $\mathrm{C}$ was termed D11S287 in accordance with the Human Gene Mapping Library system. The chromosomal mapping data show that in this tumor a major intrachromosomal recombination occurred between the PTH gene (on $11 \mathrm{p} 15$ [16]) and the D11S287 area on 11q13.

Of further interest, $11 \mathrm{q} 13$ is the location of at least three putative oncogenes, $b c l-1(8)$, int-2 (9), and hst $(18,19) . b c l-1$ is rearranged with the Ig heavy chain locus in $t(11 ; 14)$ follicular lymphomas and other hematopoietic malignancies; int- 2 is the human homologue of a mouse mammary tumor virus integration site involved in certain murine breast cancers; $h s t$ is an oncogene derived from a human gastric carcinoma, whose product may be in the fibroblast growth factor and int-2-encoded protein family. Recently, the gene for MEN-I, in which hyperparathyroidism is common, has been mapped to this region of chromosome 11 in tight linkage with the muscle

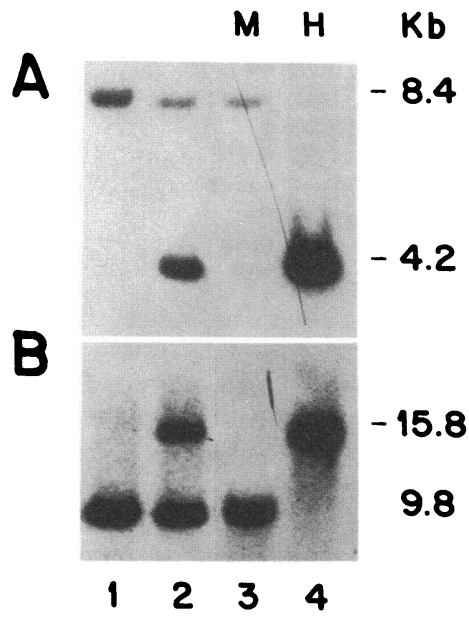

Figure 4. Southern blots of probes $\mathrm{A}$ and $\mathrm{C}$ hybridized to mouse/human cellular DNA after restriction enzyme digestion. $(A)$ Probe A hybridized to genomic DNA after Bam HI digestion. (B) Probe $C$ hybridized to DNA after Hind III digestion. Lane 4, human $(H)$ fibroblast; lane 3 , mouse $(M)$ epithelial line; lane 2, positive humanmouse cell hybrid; and lane 1 , negative human-mouse cell hybrid. 
Table I. Segregation of Probes A and C with Human Chromosomes in Human-Mouse Cell Hybrid DNA

\begin{tabular}{|c|c|c|c|c|c|c|c|c|c|c|c|c|c|c|c|c|c|c|c|c|c|c|c|}
\hline Chromosome & 1 & 2 & 3 & 4 & 5 & 6 & 7 & 8 & 9 & 10 & 11 & 12 & 13 & 14 & 15 & 16 & 17 & 18 & 19 & 20 & 21 & 22 & $X$ \\
\hline \multicolumn{24}{|l|}{ Probe A } \\
\hline Concordant no. of hybrids & 26 & 26 & 21 & 26 & 27 & 28 & 29 & 21 & 22 & 26 & 33 & 24 & 22 & 23 & 22 & 22 & 21 & 23 & 28 & 24 & 22 & 20 & \\
\hline Discordant no. of hybrids & 8 & 9 & 14 & 10 & 9 & 8 & 7 & 15 & 13 & 10 & 0 & 12 & 14 & 13 & 13 & 14 & 14 & 13 & 8 & 11 & 14 & 14 & 1 \\
\hline Probe A discordancy (\%) & 24 & 26 & 40 & 28 & 25 & 22 & 19 & 42 & 37 & 28 & 0 & 33 & 39 & 36 & 37 & 39 & 40 & 36 & 22 & 31 & 39 & 41 & \\
\hline \multicolumn{24}{|l|}{ Probe C } \\
\hline Concorda & 21 & 22 & 17 & 19 & 21 & 24 & 25 & 17 & 20 & 23 & 30 & 18 & 20 & 21 & 19 & 20 & 20 & 20 & 25 & 23 & 20 & 22 & \\
\hline Discordant no. of hybrids & 10 & 11 & 15 & 14 & 12 & 9 & 7 & 16 & 12 & 10 & 0 & 15 & 13 & 12 & 13 & 13 & 12 & 13 & 8 & 9 & 13 & 10 & \\
\hline Probe $\mathrm{C}$ discordancy (\%) & 32 & 33 & 47 & 42 & 36 & 27 & 22 & 48 & 37 & 30 & 0 & 45 & 39 & 36 & 41 & 39 & 38 & 39 & 24 & 28 & 39 & 31 & \\
\hline
\end{tabular}

The DNA probes for fragments A and C were hybridized to Southern blots containing Bam HI-digested DNA (for A) or Hind III-digested DNA $($ for $C)$ from human-mouse cell hybrids. Scoring was determined by the presence $(+)$ or absence $(-)$ of a human band in the hybrids on the blots. Fragment $A$ was scored on 36 cell hybrids involving 15 unrelated human cell lines and 4 mouse cell lines $(13,14)$. Fragment $C$ was scored on 33 cell hybrids involving 15 unrelated human cell lines and 4 mouse cell lines $(13,14)$. The hybrids in this table were characterized by chromosome analysis and mapped enzyme markers, and partly by mapped DNA probes $(13,28,29)$. The DNA fragments A and C mapped to human chromosome 11. The hybrids: EXR-5CSAz with the X/11 translocation Xpter $\rightarrow$ Xq22 :: 11q13 $\rightarrow 11$ ter and XER-7 with the $11 / \mathrm{X}$ translocation 11 qter $\rightarrow 11$ p11 $::$ Xq11 $\rightarrow$ Xqter, localize both fragment A and fragment C to the q13 $\rightarrow$ qter region of human chromosome 11.

phosphorylase gene PYGM (20). We have compared the genomic restriction maps of bcl-1 (8), int-2 (9), and hst (21) with our map of D1 1S287, and no similarities are apparent. Furthermore, neither the cloned $b c l-1$ fragment (see Methods) nor the int-2 probes SS6 and BB4 hybridized with our D11S287containing bacteriophage clones. Additionally, the int- 2 and $b c l-1$ probes did not detect rearranged bands on Southern blots of DNA from patient Y's parathyroid adenoma (nine enzymes studied). These data, of course, do not exclude the possibility that D1 1S287 is near, or even another part of, the $b c l-1$, int-2, or $h s t$ region, but are also consistent with the possibility that D11S287 is a previously uncharacterized region of the human genome.
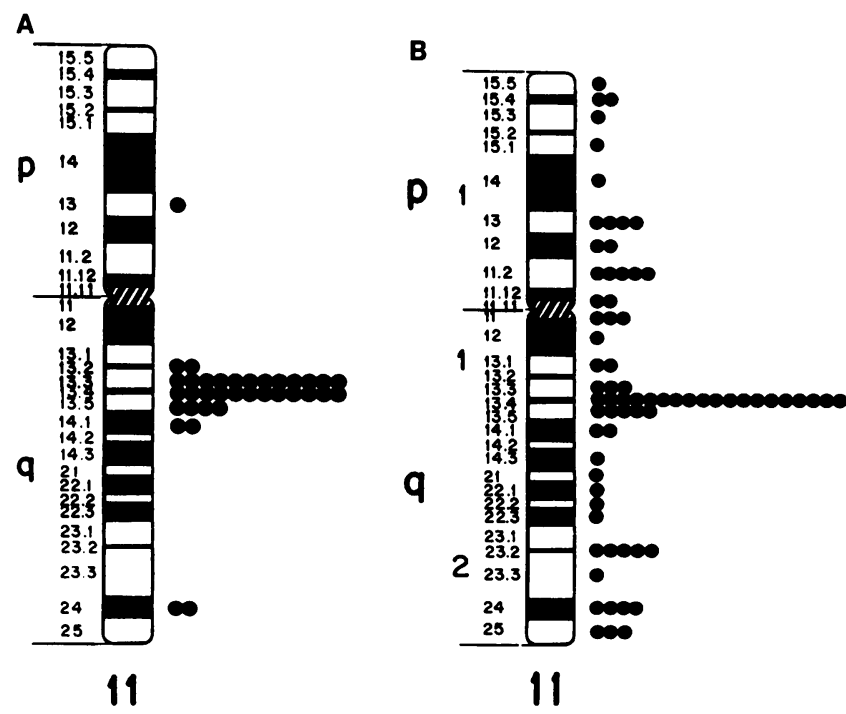

Figure 5. The distribution of grains observed on chromosome 11 for $(A)$ probe $\mathrm{A}$ and $(B)$ probe $\mathrm{C}$. For each probe, the number of metaphase cells with grains on chromosome 11 per total cells analyzed was $(A) 35 / 100$ and $(B) 72 / 100$. The total number of grains counted for $A$ was 135 and for $B 257$. There was no significant accumulation of grains above background at any other chromosomal site for each probe. D11S287 was localized to 11q13.3-q13.5.

\section{Discussion}

We have determined that the PTH locus underwent a somatic, clonal, reciprocal rearrangement with $11 \mathrm{q} 13$ in a parathyroid adenoma. This rearrangement potentially places DNA from 11 q13 under the influence of tissue-specific, active PTH regulatory elements (which could exist on $5^{\prime}$ or $3^{\prime}$ PTH regions), and could thereby result in activation of a cellular oncogene. Other mechanisms of tumorigenesis are also possible; for example, the rearrangement could have inactivated a gene on $11 \mathrm{q} 13$ that normally functions as a tumor suppressor (22). It is even conceivable that loss of one functional PTH gene per se could be linked to a stimulatory effect on parathyroid cell proliferation.

Clonal PTH gene rearrangements do not appear to be common in parathyroid adenomas; our initial survey of 43 adenomas yielded two such examples (5). This may underestimate the true prevalence of relevant PTH rearrangements, however; for example, more intensive study (with additional restriction enzymes) of five adenomas has uncovered one new clonal rearrangement in the $5^{\prime}$ PTH flanking region (unpublished observations). It is also possible that, through other mechanisms like point mutation, a gene in the D11S287 region may be involved more generally in parathyroid adenomatosis. While no restriction fragment alterations were grossly detectable with probes $\mathrm{A}, \mathrm{B}$, and $\mathrm{C}$ in six adenomas, including the other two with PTH gene alterations (unpublished observations), D1 1S287 breakpoints could certainly vary in their precise locations over substantial distances and thus be undetectable with those probes on routine Southern analyses; the c-myc breakpoints in Burkitt lymphoma, for example, may vary over a distance $>50 \mathrm{~kb}$ (1). It is of course possible that parathyroid adenomas do not share any single pathogenetic basis but, like many tumor types, are heterogeneous. Much further study will be necessary to clarify these issues. However, the PTH gene rearrangement described here, and the two others alluded to above, are likely to be important pathogenetically in the specific tumors that contain them, because they occur clonally in all the tumor cells. Furthermore, we have never observed such a rearranged DNA fragment as a low 
intensity band (i.e., present in only a minority of tumor cells) in a parathyroid adenoma or in parathyroid hyperplasia, arguing against the rearrangements being a nonspecific, stochastic event secondary to increased cell proliferation. The DNA fragments we have cloned from the breakpoint region of $11 \mathrm{q} 13$ will be important tools for the future investigation of the functional consequences of this rearrangement.

$11 \mathrm{q} 13$ is a large region on a molecular scale, and could contain several hundred genes. Thus, while the putative growth-related loci int-2, bcl-1, hst, and the MEN-I gene all map to this band and should be further investigated as being near or part of D11S287, there is no compelling reason to expect such a coincidence. DNA sequence analysis and Northern blotting of parathyroid and nonparathyroid RNA will provide initial indications of whether our D11S287 clones contain a new, possibly growth-related gene, or whether cloning of an extended stretch of genomic DNA from this region may be necessary. In vitro transformation assays could be used to further test the functional role of a candidate D1 1S287 gene. Also, a survey for restriction fragment length polymorphisms in the D11S287 region could provide new markers for genetic linkage analyses, potentially useful, for example, in the search for the MEN-I gene.

Few molecular genetic abnormalities have been previously described in benign human tumors; they include ras point mutations in colon adenomas (23) and loss of heterozygosity on chromosome 22 in meningiomas (24). Molecular evidence for clonal DNA rearrangement has not, to our knowledge, been described previously in a benign neoplasm. Moreover, the rearrangement of a gene encoding a major terminally differentiated tissue-specific protein in a tumor (benign or malignant) of a specified cell type is a novel finding, except for the Ig and $\mathrm{T}$ cell receptor genes, which rearrange physiologically. Our findings raise the possibility that analogous rearrangements may be present in other differentiated tumor types; for example, a subset of insulinomas could contain insulin gene rearrangements.

Cytogenetic studies of parathyroid tumors have not been possible and, more generally, chromosomal rearrangements have been only rarely been described in benign solid tumors (25). We have presented molecular evidence for a major aberrant chromosomal recombination in this benign parathyroid tumor, effectively bypassing the technical barrier posed by these difficulties in performing cytogenetics. Whether the cloned D11S287 DNA will be useful in detecting other rearrangements at $11 \mathrm{q} 13$ remains to be seen. A number of leukemias, for example, are known to harbor translocations involving 11q13 (26), and might be profitably studied with D1 1S287 probes. In addition, a fragile site has been found at $11 \mathrm{q} 13$ (27), and our probes could be used to assess whether the D11S287 region constitutes such a fragile site, the nature of which remains unknown on a molecular level. Whether fragile or not, determination of the nucleotide sequences adjacent to the breakpoint both in the PTH gene and in D11S287 may provide important insights into the mechanism of recombination in this and perhaps other tumors.

\section{Acknowledgments}

The authors wish to thank Dr. Clive Dickson and Dr. Yoshihide Tsujimoto for generously contributing the human int-2 and $b c l-1$ DNA probes, respectively, and Ms. Susan Rhodes and Ms. Brigid Pach for expert assistance in preparation of the manuscript.

The work was supported in part by grants DK-11794, DK-08116, and GM-20454 from the National Institutes of Health. Dr. Arnold is the recipient of a Junior Faculty Research Award from the American Cancer Society.

\section{References}

1. Croce, C. M., and P. C. Nowell. 1985. Molecular basis of human B cell neoplasia. Blood. 65:1-7.

2. Kurzrock, R., J. U. Gutterman, and M. Talpaz. 1988. The molecular genetics of Philadelphia chromosome-positive leukemias. $N$. Engl. J. Med. 319:990-998.

3. Kovacs, G., R. Erlandsson, F. Boldog, S. Ingvarsson, R. MullerBrechlin, G. Klein, and J. Sumegi. 1988. Consistent chromosome 3p deletion and loss of heterozygosity in renal cell carcinoma. Proc. Natl. Acad. Sci. USA. 85:1571-1575.

4. Whang-Peng, J., C. S. Kao-Shan, E. C. Lee, P. A. Bunn, D. N. Carney, A. F. Gazdar, and J. D. Minna. 1982. Specific chromosome defect associated with human small-cell lung cancer: deletion $3 p$ (14-23). Science (Wash. DC). 215:181-182.

5. Arnold, A., C. E. Staunton, H. G. Kim, R. D. Gaz, and H. M. Kronenberg. 1988. Monoclonality and abnormal parathyroid hormone genes in parathyroid adenomas. N. Engl. J. Med. 318:658-662.

6. Arnold, A., J. Cossman, A. Bakhshi, E. S. Jaffe, T. A. Waldmann, and S. J. Korsmeyer. 1983. Immunoglobulin-gene rearrangements as unique clonal markers in human lymphoid neoplasms. $N$. Engl. J. Med. 309:1593-1599.

7. Igarashi, T., T. Okazaki, H. Potter, R. Gaz, and H. M. Kronenberg. 1986. Cell-specific expression of the human parathyroid hormone gene in rat pituitary cells. Mol. Cell. Biol. 6:1830-1833.

8. Tsujimoto, Y., J. Yunis, L. Onorato-Showe, J. Erikson, P. C. Nowell, and C. M. Croce. 1984. Molecular cloning of the chromosomal breakpoint of B-cell lymphomas and leukemias with the $t(11 ; 14)$ chromosome translocation. Science (Wash. DC). 224:14031406.

9. Casey, G., R. Smith, D. McGillivray, G. Peters, and C. Dickson. 1986. Characterization and chromosome assignment of the human homolog of int-2, a potential proto-oncogene. Mol. Cell. Biol. 6:502510.

10. Karn, J., H. Matthes, M. Gait, and S. Brenner. 1984. A new selective phage cloning vector, $\lambda 2001$, with sites for XbaI, BamHI, HindIII, EcoRI, SstI, and Xhol. Gene (Amst.). 32:217-224.

11. Karn, J., S. Brenner, and L. Barnett. 1983. New bacteriophage lambda vectors with positive selection for cloned inserts. Methods Enzymol. 101:3-19.

12. Feinberg, A. P., and B. Vogelstein. 1983. A technique for radiolabeling DNA restriction endonuclease fragments to high specific activity. Anal. Biochem. 132:6-13.

13. Shows, T. B., A. Y. Sakaguchi, and S. L. Naylor. 1982. Mapping of the human genome, cloned genes, DNA polymorphisms, and inherited disease. Adv. Hum. Genet. 12:341-452.

14. Shows, T., R. Eddy, L. Haley, M. Byers, M. Henry, T. Fujita, H. Matsui, and T. Taniguchi. 1984. Interleukin-2 $(I L-2)$ is assigned to human chromosome 4. Somatic Cell Mol. Genet. 10:315-318.

15. Naylor, S. L., A. Y. Sakaguchi, T. B. Shows, M. L. Law, D. V. Goeddel, and P. W. Gray. 1983. Human immune-interferon gene is located on chromosome 12. J. Exp. Med. 57:1020-1027.

16. Zabel, B. U., H. M. Kronenberg, G. I. Bell, and T. B. Shows. 1985. Chromosome mapping of genes on the short arm of human chromosome 11: parathyroid hormone gene is at $11 \mathrm{p} 15$ together with the genes for insulin, c-Harvey-ras I, and $\beta$-hemoglobin. Cytogenet. Cell Genet. 39:200-205.

17. Nakai, H., M. G. Byers, T. B. Shows, and R. T. Taggart. 1986. Assignment of the pepsinogen gene complex (PGA) to human chro- 
mosome region $11 \mathrm{q} 13$ by in situ hybridization. Cytogenet. Cell Genet. 43:215-217.

18. Yoshida, T., K. Miyagawa, H. Odagiri, H. Sakamoto, P. F. R. Little, M. Terada, and T. Sugimura. 1987. Genomic sequence of $h s t$, a transforming gene encoding a protein homologous to fibroblast growth factors and the int-2-encoded protein. Proc. Natl. Acad. Sci. USA. 84:7305-7309.

19. Adelaide, J., M.-G. Mattei, I. Marics, F. Raybaud, J. Planche, O. DeLapeyriere, and D. Birnbaum. 1988. Chromosomal localization of the hst oncogene and its co-amplification with the int-2 oncogene in a human melanoma. Oncogene. 2:413-416.

20. Larsson, C., B. Skogseid, K. Oberg, Y. Nakamura, and M. Nordenskjold. 1988. Multiple endocrine neoplasia type I gene maps to chromosome 11 and is lost in insulinoma. Nature (Lond.). 332:85-87.

21. Yoshida, T., H. Sakamoto, K. Miyagawa, P. F. R. Little, M. Terada, and T. Sugimura. 1987. Genomic clone of hst with transforming activity from a patient with acute leukemia. Biochem. Biophys. Res. Commun. 142:1019-1024.

22. Friend, S. H., T. P. Dryja, and R. A. Weinberg. 1988. Oncogenes and tumor-suppressing genes. N. Engl. J. Med. 318:618-622.

23. Bos, J. L., E. R. Fearon, S. R. Hamilton, M. Verlaan-deVries,
J. H. vanBoom, A. J. van der Eb, and B. Vogelstein. 1987. Prevalence of ras gene mutations in human colorectal cancers. Nature (Lond.). 327:293-297.

24. Seizinger, B. R., S. de la Monte, L. Atkins, J. F. Gusella, and R. L. Martuza. 1987. Molecular genetic approach to human meningioma: loss of genes on chromosome 22. Proc. Natl. Acad. Sci. USA. 84:5419-5423.

25. Sandberg, A. A., and C. Turc-Carel. 1987. The cytogenetics of solid tumors. Cancer. 59:387-395.

26. Sait, S. N., P. DalCin, and A. A. Sandberg. 1987. Recurrent involvement of $11 \mathrm{q} 13$ in acute nonlymphocytic leukemia. Cancer Genet. Cytogenet. 26:351-354.

27. LeBeau, M. M. 1986. Chromosomal fragile sites and cancerspecific rearrangements. Blood. 67:849-858.

28. Shows, T. B., J. A. Brown, L. L. Haley, M. G. Byers, R. L. Eddy, E. S. Cooper, and A. P. Goggin. 1978. Assignment of the $\beta$-glucuronidase structural gene to the pter $\rightarrow$ q22 region of chromosome 7 in man. Cytogenet. Cell Genet. 21:99-104.

29. Shows, T. B. 1983. Human genome organization of enzyme loci and metabolic disease. Isozymes Curr. Top. Biol. Med. Res. 10:323-339. 